\title{
The progress of pulmonary artery denervation
}

\author{
Yonghui Xie ${ }^{1,2 *}, \mathrm{Na} \mathrm{Liu}^{3 *}$, Zhenghui Xiao $^{1}$, Fang Yang ${ }^{3}$, Yunhong Zeng ${ }^{1,2}$, \\ Zhou Yang ${ }^{1,2}$, Yuanxi Xia ${ }^{1,2}$, Zhi Chen ${ }^{1,2}$, Yunbin Xiao ${ }^{1,2}$ \\ ${ }^{1}$ Academy of Pediatrics, University of South China, Changsha, China \\ ${ }^{2}$ Department of Cardiology, Hunan Children's Hospital, Changsha, China \\ ${ }^{3}$ Department of Cardiology, The Second Xiangya Hospital of Central South University, China
}

\begin{abstract}
Pulmonary arterial hypertension (PAH) is a chronic pulmonary vascular disease characterized by increased pulmonary arterial pressure and pulmonary arterioles remodeling. Some studies have discovered the relationship between sympathetic nerves (SNs) and pathogenesis of PAH. This review is aimed to illustrate the location and components of SNs in the pulmonary artery, along with different methods and effects of pulmonary artery denervation (PADN). Studies have shown that the SNs distributed mainly around the main pulmonary artery and pulmonary artery bifurcation. And the SNs could be destroyed by three ways: the chemical way, the surgical way and the catheter-based way. PADN can significantly decrease pulmonary arterial pressure rapidly, improve hemodynamic varieties, and then palliate PAH. PADN has been recognized as a prospective and effective therapy for PAH patients, especially for those with medication-refractory $P A H$. However, further enlarged clinical studies are needed to confirm accurate distribution of SNs in the pulmonary artery and the efficacy of PADN. (Cardiol J 2022; 29, 3: 381-387)
\end{abstract}

Key words: pulmonary arterial hypertension, pulmonary artery denervation, sympathetic nerves

\section{Introduction}

Pulmonary arterial hypertension (PAH), characterized by mean pulmonary artery pressure (mPAP) $\geq 25 \mathrm{mmHg}$ at rest, pulmonary artery wedge pressure (PAWP) $\leq 15 \mathrm{mmHg}$ and pulmonary vascular resistance (PVR) $>3$ Wood units [1], is a disease induced by a wide range of causes, which makes it difficult to formulate an appropriate therapeutic plan and receive great responses for $\mathrm{PAH}$ patients [2]. Up till the present, most patients can only rely on combined targeted medicine, however, not all patients can be relieved [2,3]. Besides, the 1-year, 3 -year and 5-year survival rate of medium to high- -risk patients treated by target therapy are $90 \%, 61 \%$, $43 \%$ [4], and side effects of targeted medicine are not tolerable in a considerable number of patients. Thus, these patients are eager to acquire better therapies. Recently, pulmonary artery denervation (PADN), as a novel therapy, has gradually proved beneficial to improve hemodynamic measurements in animal models and in $\mathrm{PAH}$ patients through regulating the autonomic nervous system [5]. Previous studies have proved that PADN can significantly decrease pulmonary arterial pressure rapidly, and then palliate the development of PAH through destroying the sympathetic nerves (SNs) of the pulmonary artery (PA) [6].

Address for correspondence: Yunbin Xiao, PhD, MD, Department of Cardiology, Hunan Children's Hospital, No. 86 Ziyuan Road, Yuhua District, Changsha, Hunan, 410007, China, tel: +8673185356800, +8613549658136, fax: (0731)5356666, e-mail: xiaoyunbinrui@126.com

Received: 27.10.2020 Accepted: 6.12.2020 Early publication date: 31.12 .2020

*Equal contributors.

This article is available in open access under Creative Common Attribution-Non-Commercial-No Derivatives 4.0 International (CC BY-NC-ND 4.0) license, allowing to download articles and share them with others as long as they credit the authors and the publisher, but without permission to change them in any way or use them commercially. 


\section{Sympathetic nerves along the pulmonary artery}

The autonomic nervous system consists of sympathetic and parasympathetic parts, which are regulated by various neural and endocrine factors [7]. The sympathetic nervous system (SNS) arises from the spinal cord (T1-L2), and postganglionic neurons administrate the pulmonary vascular systems $[7,8]$. Studies have proved that the components of SNs around PA include many tyrosine hydroxylase (TH)-positive, neuropeptide-Y (NPY)-positive and a few calcitonin-gene-related peptide (CGRP)-positive nerve fibers in rat models and in humans [9]. Thus, when SNS is activated, the SNs can locally release neurohormones to enhance the vascular tone so that the PA pressure will be quickly elevated. Furthermore, chronic inflammation is known as a critical factor contributing to the progress of PAH [10], and de Juan et al. [11] has investigated that sympathetic nerves of arteries play an important role in inflammation. Therefore, the over-activation of SNS [12] may accelerate the development of $\mathrm{PAH}$ via regulating the inflammation.

Rothman et al. [13] deemed that SNs around the main pulmonary artery (MPA) and PA bifurcation were relatively larger than those around the left pulmonary artery (LPA) or right pulmonary artery (RPA). Although there is not enough evidence about the distribution of SNs around PA in humans, most animal studies also represented that SNs distributed mostly around MPA and PA bifurcation [9, 14-16], while diverse races may have a little difference in the distribution site of SNs. Currently, many animals can be used as PAH models, and mainly include two animals. Big animals, including swine [16, 17], dogs [14, 15], sheep [18], rabbits [19] and so on, which are easier to operate on because of their larger vessels, better tolerance to surgical injury, higher degree of the similarity to humans, but the cost in time and money is also much higher. Small animals, such as mice and rats $[9,20]$, are more suitable for surgical models, because they can recover quickly and a have stronger anti-infection ability, but skilled operators are needed.

Heretofore, some studies have shown the distribution of SNs around the PA in swine, dog and rat models (Fig. 1). Rothman et al. [16] showed that most nerves are located approximately 1 to $3 \mathrm{~mm}$ from the luminal aspect in the MPA of swine. Besides, the diameter of SNs is greater than those in the distal PA. In the LPA and RPA, almost all

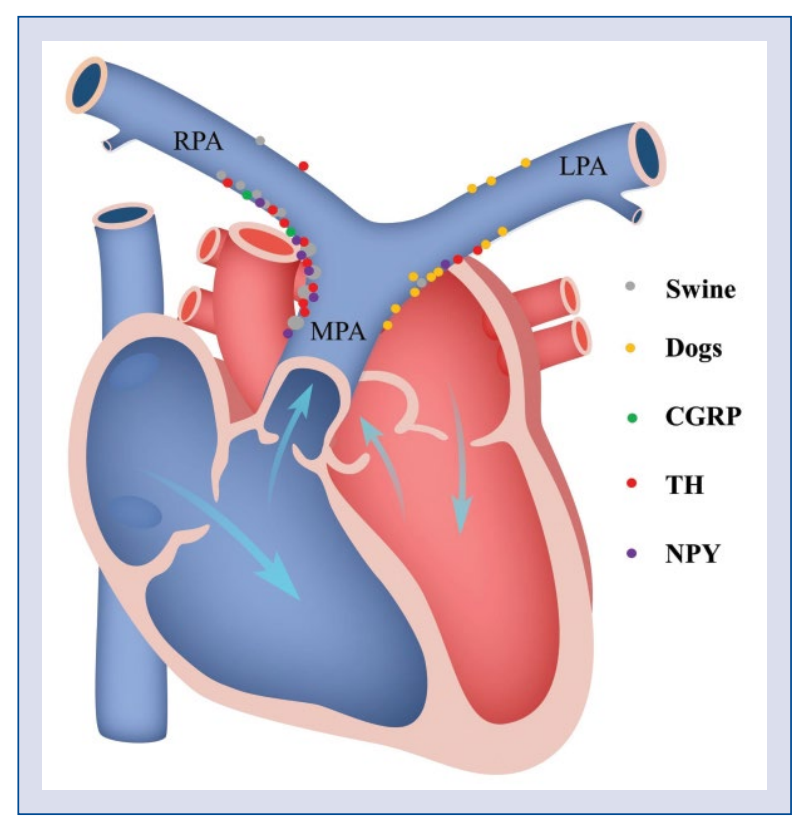

Figure 1. Distribution of sympathetic nerves (SNs) of pulmonary artery (PA) in different races. In dogs, most nerves (yellow spots) are distributed in posterior wall of main pulmonary artery (MPA) and left pulmonary artery (LPA), while in swine (gray spots), the SNs are distributed mostly in posterior and right of MPA. Besides, in PA of rats, the components of SNs around PA include many TH-positive nerve fibers (red spots), NPY-positive nerve fibers (purple spots) and a few CGRP-positive nerve fibers (green spots). TH - tyrosine hydroxylase; NPY - neuropeptide-Y; CGRP - calcitonin-gene-related peptide; RPA — right pulmonary artery.

nerves are located less than $1 \mathrm{~mm}$ away from intraartery surface. There are two distal boundaries, including the ostium of left posterior artery and right posterior descending artery. The nerve bundles are located in the posterior and right of MPA and posterior and lateral of the RPA [16]. But in dogs, Zhou et al. [15] reported that PA sympathetic nerve bundles are located in the left of MPA, and most branches were distributed in the posterior wall of MPA and LPA, which is different from the distribution of SNs in swine. Furthermore, Huang et al. [9] investigated that the distribution of nerves was mainly in the adipose and connective tissues in rats, especially located in posterior and right anterior of MPA and PA bifurcation, which was the same with the distribution of SNs in swine. Compared with normal rats, the area of SNs distribution was significantly larger in the PAH rats, and the SNs of PAH rats mainly located in posterior and right anterior of MPA and PA bifurcation. 


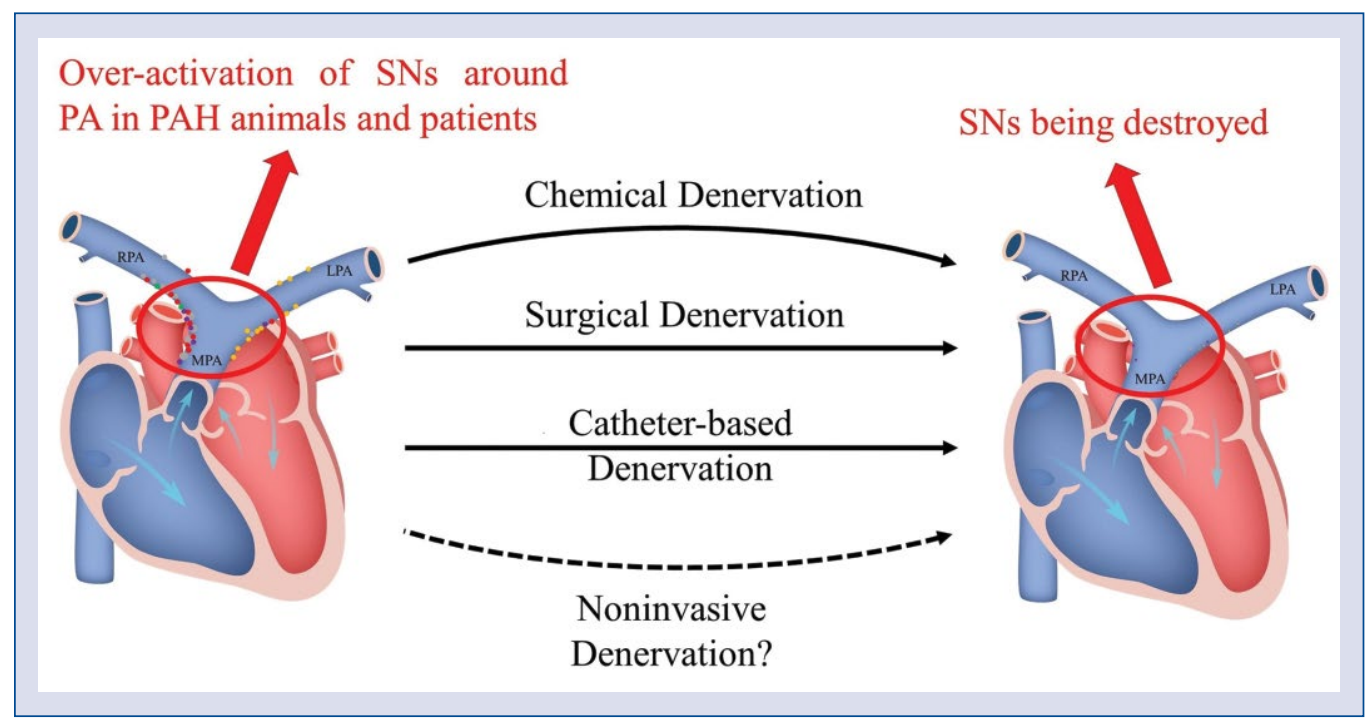

Central illustration. Schematic diagram summarizing principles and methods of pulmonary artery denervation (PADN). The area of sympathetic nerves (SNs) distribution was significantly larger in the pulmonary arterial hypertension (PAH) animals and patients. PADN can destroy those SNs and then palliate PAH; PA — pulmonary artery.

\section{Pulmonary artery denervation}

Pulmonary artery denervation is aimed at destroying the anatomic structures and functions of SNs along the PA by three methods, including chemical denervation, surgical denervation and catheter-based denervation. Since 1980, Juratsch et al. [21] found that elevation of mPAP increased by balloon distension of MPA can be abolished by destroying SNs distributed around PA bifurcation via surgical or chemical measures [21]. From then on, more and continuing studies are conducted to search for the best way to perform PADN (Central illustration).

\section{Chemical denervation}

Chemical denervation is performed via using the SNs blocking drugs to damage nerves around PA by intravenous infusion [21] or intraperitoneal injection [22]. To date, there have only been a few animal studies referring to chemical PADN, most of the studies used 6-hydroxydopamine (6-OHDA), a mediator of adrenergic nerves, to destroy the activity of SNs [22]. Juratsch et al. [21] has performed chemical denervation with 8 dogs, and then compared the increments of mPAP post balloon inflation in MPA. After destroying the pulmonary adrenergic nerves, the responses to balloon inflation in MPA was relieved, and increased the value of pulmonary arterial pressure (PAP) and PVR all declined markedly when compared with before denervation in these dogs [21]. There is no doubt that this way is the most convenient, while the side effects cannot be ignored. Jiang et al. [22] has shown that chemical sympathectomy with administration of 6-OHDA caused dysregulation of the cardiac autonomic nervous system and myocardial injury. Moreover, administration of 6-OHDA can produce selective degeneration of adrenergic nerve terminals and blockades or destroying adrenergic receptor sites [23] which consisted of the pathogenesis of diabetes mellitus. Thus, it is necessary to find a new drug, which is only plays a role in SNs around PA, or find a new way to make the drug to only influence PA nerves. Recently, there have been many studies trying to perform denervation with magnetic nanoparticles (MNP) [24]. Yu et al. [25] has done autonomic denervation in four major atrial ganglionated plexi (GP) suppressed atrial fibrillation with MNP. They also successfully ablated GP by external electromagnet pulled Ca-MNP to the targeted GP after an injected Ca-MNP in the coronary artery [26]. Thus, further studies may conduct an active targeting magnetic nanoparticle with 6-OHDA, then using magnetic material in vitro guiding MNP with 6-OHDA in MPA and PA bifurcation. Since aminopeptidase P (APP) has been confirmed specifically distributed in PA endothelium [27], an active targeting drug delivery system can be considered, such as APP antibody-decorated nanoparticles with 6-OHDA loading. 


\section{Surgical denervation}

With further research being conducted, the distribution of SNs has been clearly depicted that many nerves located in the adipose and connective tissues of PA adventitia [28]. The performance of surgical denervation is mainly via removing adipose and connective tissues of PA adventitia. Juratsch et al. [21] performed surgical denervation in dogs with elevated PAP when the balloon was inflated in the MPA. After denervation, both systolic and diastolic pulmonary artery pressure of these dogs did not significantly change when the balloon inflated. Transthoracic pulmonary artery denervation (TPADN) is more accurate and direct, after thoracotomy in the left third intercostal space, the adipose and connective tissues around the trunk and branches of MPA were exfoliated using microsurgical techniques. The main PA trunk and bifurcation and the proximal regions of the LPA and RPA were the key stripping areas for TPADN. Compared with the sham group, the mPAP of the test group decreased significantly after denervation. Furthermore, they demonstrated that the muscularization rate declined after denervation [9]. Although TPADN was a more effective way to reduce $\mathrm{mPAP}$ in monocrotaline $\mathrm{PAH}$ rat models, still the largest limitation cannot be ignored that the surgery itself is an invasive way with high risks, especially for those who cannot afford anesthesia, which makes it very difficult to apply for PAH patients, so a less invasive PADN method is needed.

\section{Catheter-based denervation}

Catheter-based denervation is already applied to PAH patients for its micro-traumatic, low-risk and quick effect. Many animal studies and clinical studies have proved the curative effect of ablation [29]. After inserting a sheath through femoral vein and advancing to MPA and PA bifurcation after PA angiography, then the PADN catheter was advanced along this long sheath. Letting the circular tip place in the lumen of MPA and PA bifurcation where SNs were mostly distributed [30], then ablation was conducted. For most animals and PAH patients, the MPAP and PVR were decreased immediately to some degree and were declining in the follow-up months after PADN. There was some catheterbased PADN conducted in swine [16] and dogs [15], and these studies found that the SNs injury induced by PADN could remain for a long time, and PADN continually improved hemodynamic parameters and PA remodeling in PAH animal models $[15,16]$. To date, catheter-based PADN has been applied in clinical trials. Chen et al. [30] did the first PADN clinical study with low-risk idiopathic pulmonary arterial hypertension (IPAH) patients, and showed that the PADN procedure can improve hemodynamic measurements and clinical presentation quickly and effectively. In this trial, the systolic PAP and mPAP of 12 patients with PADN procedures obviously declined at once and during the subsequent 3 months. Furthermore, during the 3-month follow-up, these IPAH patients who performed PADN procedures showed a significant improvement in cardiac output and 6 -min walk distance (6WMD). In the treatment group, just one procedure failed when the patient suddenly experienced intolerable chest pain after the firstplace ablation (three ablation places in total), but mPAP still reduced $6 \mathrm{mmHg}$ [30]. Although this study has investigated hemodynamic improvements and functional capacity of IPAH patients after PADN, the conclusion that PADN was an effective therapy for medication-refractory PAH patients was still recognized with controversies in the low-risk type and insufficient quantity of PAH patient individuals for nonrandomized trials, no sham populations and not enough follow-up. To confirm the efficacy of PADN, Chen et al. then performed other studies that enrolled varieties of patients from different centers, in different World Health Organization groups and were induced by different causes [31-33], thereafter they found that performing PADN in the internal surface of PA can still achieve a significant curative effect for those PAH patients who can bear chest pain induced by the PADN procedure after ablation. However, there are still some patients who cannot achieve benefits from this type of PADN. It is probably associated with the limited depth of ablation in the lumen and only a few SNs being destroyed. Moreover, the TIVUS system, a novel ultrasound energy delivery system using a multidirectional ultrasound catheter, is being observed because it can prolong the depth of ablation. Rothman et al. [34] lately conducted related animal and clinical experiments, and manifested that PADN using catheter-based ultrasonic energy can also improve states of pulmonary hypertension $(\mathrm{PH})$ in animal models and $\mathrm{PAH}$ patients. In addition, studies showed that many SNs exist in the PA adventitia. And Garcia-Lunar et al. [35] conducted an animal trial using a bipolar radiofrequency system to perform PADN in the PA adventitia after thoracotomy, but hemodynamic parameters of the animal models did not improve significantly after PADN. Recently, Mahfoud et al. [36] reported an innovative alcohol-mediated renal denervation system which could infuse absolute 
alcohol through needles being penetrated into the renal artery and safely decrease blood pressure in patients. Similarly, this system also can be used in PADN so that drugs can be injected to destroy the SNs in the whole layer of PA, and then make most SNs around PA inactive aiming to minimize the over-activity of SNS and rein-angiotensin-aldosterone system (RAAS) in a larger extent.

\section{Changes of sympathetic nerves after pulmonary artery denervation}

After PADN, the anatomic structure and functions of SNs around PA both have been destroyed to some extent, though PADN itself cannot destroy all SNs. Early preclinical studies have shown that sympathetic nerve conduction velocity (SNCV) immediately changed when PADN was done. In a study with 41 dogs ( 6 control, 10 with mPAP $\leq 25 \mathrm{mmHg}$ after injected dehydrogenized monocrotaline (DHMCT), 5 with PAH but without PADN, 20 with PADN) was used to examine SNs, the 20 PAH dogs induced by DHMCT underwent PADN at the conjunctional area between the distal MPA and the ostial LPA with the following parameters: a temperature of $45^{\circ}$ to $50^{\circ}$, energy $\leq 10 \mathrm{~W}$, and times of $120 \mathrm{~s}$. Compared with the prior-PADN groups, the SNCV of those with $\mathrm{mPAP} \geq 25 \mathrm{mmHg}$ was decreased immediately after the PADN procedure. Furthermore, in the follow-up during 3 months, SNCV was continually declining and eventually almost equalled the control animals. Nevertheless, in the study, the axon diameter and myelin thickness of these dogs with mPAP $\geq 25 \mathrm{mmHg}$ was noticeably higher than those with $\mathrm{mPAP} \leq 25 \mathrm{mmHg}$. Compared with the sham group, the axon diameter and myelin thickness had a gradual decline in the PADN group post PADN. The same with SNCV, in the subsequent 3 months, the axon diameter was decreasing, and the myelin sheath was increasingly thinner over time. Eventually, the nerves became almost demyelinated [15]. These findings represented that PADN can induce serious and long-term SNs injury and then decrease the activity of SNS [6]. Furthermore, some studies reported that in the lung tissue, main components of RAAS are decreased in the PADN group [37], such as renin, angiotensin converting enzyme (ACE), angiotensin II (Ang II), Ang II type 2 receptor (AT2 receptor), mineralocorticoid receptor (MR) and Ang II type 1 receptor (AT1 receptor), which illustrated that the activity of RAAS was reduced after SNs were destroyed.

\section{Improvements of hemodynamic measurements after pulmonary artery denervation}

\section{Animal studies}

Pulmonary artery denervation can almost safely and effectively abolish the increases of $\mathrm{mPAP}$ in PAH animals induced by a variety of measures, including using balloon occlusion at the interlobar segment [38], injecting DHMCT $[9,15]$ and infusing thromboxane A2 [16]. These studies have illustrated that PADN can decrease mPAP immediately and mPAP continued to decline in the following minimum of 3 months. Eventually, the $\mathrm{mPAP}$ of these PAH models with PADN procedures may even be similar to the normal animals. Zhou et al. [15] examined the hemodynamic changes of dogs found that compared with the sham group, mPAP, right atrial pressure (RAP), systolic PAP, and PVR were all significantly reduced and cardiac output was improved in PAH dogs after the PADN procedure. Zhang et al. [39] also investigated that PADN is effective in the PAH rat models secondary to heart failure, which is a disease without a useful therapy at present.

\section{Clinical studies}

At present, only catheter-based denervation is applied to clinical studies, and has achieved great effect similar to animal studies. PADN has been applied to many types of PAH patients, including IPAH [30], PAH secondary to connective diseases, PAH associated with congenital heart diseases [31], combined pre- and post-capillary $\mathrm{PH}$ associated with left heart failure [32] and so on. For most patients, PADN can quickly and significantly improve hemodynamic measurements, clinical symptoms, functional capacity and clinical follow-up. These trials all indicated that PADN is a prospective therapy for PAH patients, but a few patients cannot tolerate PADN procedures for unbearable chest pain and some patients seem to be irresponsive to PADN. Studies reported that there are two key adrenergic receptors (ARs) in PA, namely, $\beta$-ARs and $\alpha 1$-ARs. PADN can downregulate the density of $\alpha 1$-ARs and upregulate $\beta$-ARs in lung tissues and then improve PA contraction and remodeling [39]. In these patients with little benefit from PADN, perhaps it was related to the capacity of regulating ARs being lost, then the constriction of PA cannot be mediated. Moreover, Tzafriri et al. [40] found that arterial microanatomy could influence effect of catheter-based renal denervation. They 
demonstrated that power density did not peak at the arterial lumen interface where the electrode was located, and surrounding tissues, especially in water-rich tissues, like lymph nodes, could change power density diffusing to a certain extent. Furthermore, blood vessels adjacent to electrode could clear power density to a great extent. Similarly, for those patients without benefits from PADN, perhaps this phenomenon was owing to those surrounding tissues and vessels around the electrode, which changes power density diffusing, and then the degree of SNs destruction is insufficient [40]. In addition, Fujisawa et al. [41] performed PADN successfully after determining ablation sites through observing the patient's responses when high-output burst electric stimulation was applied to PA, which made the ablation sites more appropriate and avoided damaging other important nerves at the same time. Thus, to achieve better benefits, PADN should be conducted individually and clinical studies still have a long way to go.

\section{Conclusions}

In conclusion, PADN is a prospective and effective medical therapy for $\mathrm{PAH}$ patients, especially for those with medication-refractory PAH or PAH patients with limited treatment options, such as combined pre- and post-capillary PH [42]. While the mechanisms of PADN are still equivocal. In addition, there are still many issues which are eager to be resolved: (1) Previous studies have mostly investigated the distribution, components of SNs in animal models, and there are insufficient studies with humans. (2) which layer is more suitable for conducting PADN? The adventitia? The inner surface? Or all the layers? (3) Is TPADN, catheter-based denervation or accurate drug-based ablation the best method? And there already exist newmethods for renal denervation [43], such as alcohol-mediated denervation, ultrasonic energybased ablation [44] and noninvasive stereotactic body radiotherapy [45], which brings a new direction in performing PADN. (4) The long-term efficacy, the indications and contraindications are still indeterminate. These questions should be answered by further studies.

\section{Funding}

This work was supported by Hunan Provincial Key Laboratory of Emergency Medicine for Children (grant 2018TP1028), Project of Hunan Provincial Research on Chinese Medicine (grant 201914), Hunan Provincial Natural Science Youth
Foundation of China (grant 2019JJ50881), Hunan Provincial Innovation Foundation For Postgraduate (grant CX20200984) and Hunan Provincial Health Commission project (grant 20200587 and 20200596).

\section{Conflict of interest: None declared}

\section{References}

1. Galiè N, Humbert M, Vachiery J-L, et al. 2015 ESC/ERS Guidelines for the diagnosis and treatment of pulmonary hypertension. Eur Heart J. 2016; 37: 67-119, doi: 10.1093/eurheartj/ehv317, indexed in Pubmed: 26320113.

2. Barberà JA, Román A, Gómez-Sánchez MÁ, et al. Guidelines on the diagnosis and treatment of pulmonary hypertension: summary of recommendations. Arch Bronconeumol. 2018; 54(4): 205-215, doi: 10.1016/j.arbres.2017.11.014, indexed in Pubmed: 29472044.

3. Leopold JA. Catheter-based therapies for patients with medication-refractory pulmonary arterial hypertension. Circ Cardiovasc Interv. 2015; 8(11): e003332, doi: 10.1161/CIRCINTERVENTIONS.115.003332, indexed in Pubmed: 26553702.

4. Hoeper MM, Pittrow D, Opitz C, et al. and Huscher D. Risk assessment in pulmonary arterial hypertension. Eur Respir J. 2018; 51.

5. Le T, Makar C, Morway P, et al. Pulmonary artery denervation: a novel treatment modality for pulmonary hypertension. J Thorac Dis. 2019; 11(4): 1094-1096, doi: 10.21037/jtd.2019.02.93, indexed in Pubmed: 31179049.

6. Kim CW, Aronow WS, Dutta T, et al. Pulmonary artery denervation as an innovative treatment for pulmonary hypertension with and without heart failure. Cardiol Rev. 2020 [Epub ahead of print], doi: 10.1097/CRD.0000000000000299, indexed in Pubmed: 32032132.

7. Vaillancourt M, Chia P, Sarji S, et al. Autonomic nervous system involvement in pulmonary arterial hypertension. Respir Res. 2017; 18(1): 201, doi: 10.1186/s12931-017-0679-6, indexed in Pubmed: 29202826.

8. Maron BA, Leopold JA. Emerging concepts in the molecular basis of pulmonary arterial hypertension: part II: neurohormonal signaling contributes to the pulmonary vascular and right ventricular pathophenotype of pulmonary arterial hypertension. Circulation. 2015; 131(23): 2079-2091, doi: 10.1161/CIRCULATIONAHA.114.006980, indexed in Pubmed: 26056345.

9. Huang Y, Liu YW, Pan HZ, et al. Transthoracic pulmonary artery denervation for pulmonary arterial hypertension. Arterioscler Thromb Vasc Biol. 2019; 39(4): 704-718, doi: 10.1161/ATVBAHA.118.311992, indexed in Pubmed: 30816802.

10. Thenappan T, Ormiston ML, Ryan JJ, et al. Pulmonary arterial hypertension: pathogenesis and clinical management. BMJ. 2018; 360: j5492, doi: 10.1136/bmj.j5492, indexed in Pubmed: 29540357.

11. de Juan A, Ince LM, Pick R, et al. Artery-Associated sympathetic innervation drives rhythmic vascular inflammation of arteries and veins. Circulation. 2019; 140(13): 1100-1114, doi: 10.1161/CIRCULATIONAHA.119.040232, indexed in Pubmed: 31401849.

12. Velez-Roa S, Ciarka A, Najem B, et al. Increased sympathetic nerve activity in pulmonary artery hypertension. Circulation. 2004; 110(10): 1308-1312, doi: 10.1161/01. CIR.0000140724.90898.D3, indexed in Pubmed: 15337703.

13. Rothman A, Jonas M, Castel D, et al. Pulmonary artery denervation using catheter-based ultrasonic energy. EuroIntervention. 2019; 15(8): 722-730, doi: 10.4244/EIJ-D-18-01082, indexed in Pubmed: 31062694. 
14. Zhang Y, Chen W, Xu Y, et al. Nerve distribution of canine pulmonary arteries and potential clinical implications. Am J Transl Res. 2016; 8: 365-374.

15. Zhou L, Zhang J, Jiang XM, et al. Pulmonary artery denervation attenuates pulmonary arterial remodeling in dogs with pulmonary arterial hypertension induced by dehydrogenized monocrotaline. JACC Cardiovasc Interv. 2015; 8(15): 2013-2023, doi: 10.1016/j.jcin.2015.09.015, indexed in Pubmed: 26738673.

16. Rothman AMK, Arnold ND, Chang W, et al. Pulmonary artery denervation reduces pulmonary artery pressure and induces histological changes in an acute porcine model of pulmonary hypertension. Circ Cardiovasc Interv. 2015; 8(11): e002569, doi: 10.1161/CIRCINTERVENTIONS.115.002569, indexed in Pubmed: 26553697.

17. Watanabe S, Ishikawa K, Plataki M, et al. Safety and longterm efficacy of AAV1.SERCA2a using nebulizer delivery in a pig model of pulmonary hypertension. Pulm Circ. 2018; 8(4): 2045894018799738, doi: 10.1177/2045894018799738, indexed in Pubmed: 30129881.

18. Zhou X, Wang D, Castro CY, et al. A pulmonary hypertension model induced by continuous pulmonary air embolization. J Surg Res. 2011; 170(1): e11-e16, doi: 10.1016/j.jss.2011.04.030, indexed in Pubmed: 21696769.

19. Wang W, Liu R, Cao G, et al. A reliable rabbit model for hyperkinetic pulmonary hypertension. J Thorac Cardiovasc Surg. 2010; 140(2): 395-399, doi: 10.1016/j.jtcvs.2009.04.071, indexed in Pubmed: 20570291.

20. Jungebluth P, Ostertag H, Macchiarini P. An experimental animal model of postobstructive pulmonary hypertension. J Surg Res. 2008; 147(1): 75-78, doi: 10.1016/j.jss.2007.05.042, indexed in Pubmed: 18262548 .

21. Juratsch CE, Jengo JA, Castagna J, et al. Experimental pulmonary hypertension produced by surgical and chemical denervation of the pulmonary vasculature. Chest. 1980; 77(4): 525-530, doi: 10.1378/chest.77.4.525, indexed in Pubmed: 7357977.

22. Jiang YH, Jiang P, Yang JL, et al. Cardiac dysregulation and myocardial injury in a 6-hydroxydopamine-induced rat model of sympathetic denervation. PLoS One. 2015; 10(7): e0133971, doi: 10.1371/journal.pone.0133971, indexed in Pubmed: 26230083.

23. Kostrzewa RM, Jacobowitz DM. Pharmacological actions of 6-hydroxydopamine. Pharmacol Rev. 1974; 26(3): 199-288, indexed in Pubmed: 4376244.

24. Kraitchman DL, Bulte JWM. Magnetic nanoparticles and neurotoxins for treating atrial fibrillation: a new way to get burned? Circulation. 2010; 122(25): 2642-2644, doi: 10.1161/CIRCULATIONAHA.110.000166, indexed in Pubmed: 21135362.

25. Yu L, Scherlag BJ, Dormer K, et al. Autonomic denervation with magnetic nanoparticles. Circulation. 2010; 122(25): 2653-2659, doi: 10.1161/CIRCULATIONAHA.110.940288, indexed in Pubmed: 21135360 .

26. Yu L, Scherlag BS, Dormer K, et al. Targeted ganglionated plexi denervation using magnetic nanoparticles carrying calcium chloride payload. JACC Clin Electrophysiol. 2018; 4(10): 1347-1358, doi: 10.1016/j.jacep.2018.06.012, indexed in Pubmed: 30336881.

27. Chrastina A, Valadon P, Massey KA, et al. Lung vascular targeting using antibody to aminopeptidase P: CT-SPECT imaging, biodistribution and pharmacokinetic analysis. J Vasc Res. 2010; 47(6): 531-543, doi: 10.1159/000313880, indexed in Pubmed: 20431301.

28. Ogo T. Transthoracic pulmonary artery denervation. Arterioscler Thromb Vasc Biol. 2019; 39(6): 979-981, doi: 10.1161/ATVBAHA.119.312591, indexed in Pubmed: 31116607.

29. Zhang H, Chen SL. Pulmonary artery denervation: update on clinical studies. Curr Cardiol Rep. 2019; 21(10): 124, doi: 10.1007/s11886-019-1203-z, indexed in Pubmed: 31486924.

30. Chen SL, Zhang FF, Xu J, et al. Pulmonary artery denervation to treat pulmonary arterial hypertension. J Am Coll Cardiol. 2013; 62(12): 1092-1100, doi: 10.1016/j.jacc.2013.05.075.

31. Chen SL, Zhang H, Xie DJ, et al. Hemodynamic, functional, and clinical responses to pulmonary artery denervation in patients with pulmonary arterial hypertension of different causes: phase II results from the Pulmonary Artery Denervation-1 study. Circ Cardiovasc Interv. 2015; 8(11): e002837, doi: 10.1161/CIRCINTERVENTIONS.115.002837, indexed in Pubmed: 26553699.

32. Zhang $\mathrm{H}$, Zhang J, Chen $\mathrm{M}$, et al. Pulmonary artery denervation significantly increases 6-min walk distance for patients with combined pre- and post-capillary pulmonary hypertension associated with left heart failure: the PADN-5 study. JACC Cardiovasc Interv. 2019; 12(3): 274-284, doi: 10.1016/j.jcin.2018.09.021, indexed in Pubmed: 30732732.

33. Zhang H, Zhang J, Xie DJ, et al. Pulmonary artery denervation for treatment of a patient with pulmonary hypertension secondary to left heart disease. Pulm Circ. 2016; 6(2): 240-243, doi: 10.1086/685550, indexed in Pubmed: 27252851.

34. Rothman AMK, Vachiery JL, Howard LS, et al. Intravascular Ultrasound Pulmonary Artery Denervation to Treat Pulmonary Arterial Hypertension (TROPHY1): Multicenter, Early Feasibility Study. JACC Cardiovasc Interv. 2020; 13(8): 989-999, doi: 10.1016/j.jcin.2019.12.027, indexed in Pubmed: 32327095.

35. Garcia-Lunar I, Pereda D, Santiago E, et al. Effect of pulmonary artery denervation in postcapillary pulmonary hypertension: results of a randomized controlled translational study. Basic Res Cardiol. 2019; 114(2): 5, doi: 10.1007/s00395-018-0714-x, indexed in Pubmed: 30635778.

36. Mahfoud F, Renkin J, Sievert H, et al. Alcohol-Mediated renal denervation using the peregrine system infusion catheter for treatment of hypertension. JACC Cardiovasc Interv. 2020; 13(4): 471-484, doi: 10.1016/j.jcin.2019.10.048, indexed in Pubmed: 32081241.

37. Liu C, Jiang XM, Zhang J, et al. Pulmonary artery denervation improves pulmonary arterial hypertension induced right ventricular dysfunction by modulating the local renin-angiotensinaldosterone system. BMC Cardiovasc Disord. 2016; 16(1): 192, doi: 10.1186/s12872-016-0366-4, indexed in Pubmed: 27724864.

38. Chen SL, Zhang YJ, Zhou L, et al. Percutaneous pulmonary artery denervation completely abolishes experimental pulmonary arterial hypertension in vivo. EuroIntervention. 2013; 9(2): 269 -276, doi: 10.4244/EIJV9I2A43, indexed in Pubmed: 23466961.

39. Zhang H, Yu W, Zhang J, et al. Pulmonary artery denervation improves hemodynamics and cardiac function in pulmonary hypertension secondary to heart failure. Pulm Circ. 2019; 9(2): 2045894018816297, doi: 10.1177/2045894018816297, indexed in Pubmed: 30421645.

40. Tzafriri AR, Keating JH, Markham PM, et al. Arterial microanatomy determines the success of energy-based renal denervation in controlling hypertension. Sci Transl Med. 2015; 7(285): 285ra65, doi: 10.1126/scitranslmed.aaa3236, indexed in Pubmed: 25925684.

41. Fujisawa T, Kataoka M, Kawakami T, et al. Pulmonary artery denervation by determining targeted ablation sites for treatment of pulmonary arterial hypertension. Circ Cardiovasc Interv. 2017; 10(10): e006244, doi: 10.1161/CIRCINTERVENTIONS.117.005812, indexed in Pubmed: 29042401.

42. Dimopoulos K, Ernst S, McCabe C, et al. Pulmonary artery denervation: a new, long-awaited interventional treatment for combined pre- and post-capillary pulmonary hypertension? JACC Cardiovasc Interv. 2019; 12(3): 285-288, doi: 10.1016/j. jcin.2018.10.045, indexed in Pubmed: 30732733.

43. Bhatt DL, Majithia A. Continued evolution of renal artery denervation for hypertension. JACC Cardiovasc Interv. 2020; 13(4): 485-487, doi: 10.1016/j.jcin.2019.12.002, indexed in Pubmed: 32081242.

44. Kjeldsen SE, Narkiewicz K, Burnier M, et al. Renal denervation achieved by endovascular delivery of ultrasound in RADIANCE-HTN SOLO or by radiofrequency energy in SPYRAL HTN-OFF and SPYRAL-ON lowers blood pressure. Blood Press. 2018; 27(4): 185-187, doi: 10.1080/08037051.2018.1486178, indexed in Pubmed: 29936868.

45. Cai X, Yang Y, Shen Y, et al. Noninvasive stereotactic radiotherapy for renal denervation in a swine model. J Am Coll Cardiol. 2019; 74(13): 1697-1709, doi: 10.1016/j.jacc.2019.07.053, indexed in Pubmed: 31558254. 